\title{
I fanghi prodigiosi. Gli studi naturalistici sulle salse emiliane
}

\author{
Francesco Luzzini \\ Università degli Studi di Milano - Dipartimento di Bioscienze \\ francesco_luzzini@yahoo.com
}

«Il fango di questa salsa da' chirurghi, e medici reggiani viene prescritto, per corroborare le parti languide del nostro corpo, per discutere tumori edematosi, o linfe stagnanti: e intese il Sig. Vallisneri da que' paesani, che con molto frutto anche nelle risipole se ne servivano. I rognosi bagnandosi con quell'acqua certamente risanano, e molti da ulcere fetide sono perfettamente guariti» (Vallisneri 1728).

Nell'apprendere che il «fango» di cui sopra è in realtà una miscela d'argilla, acqua, anidride carbonica, metano e altre sostanze bituminose (fra cui una percentuale pericolosamente significativa di petrolio), più d'un lettore odierno storcerebbe il naso di fronte all'entusiasmo con cui, fra XV e XVIII secolo, se ne magnificavano le proprietà guaritrici. Ma la storia della medicina insegna a non stupirsi troppo di certe stranezze. Sfogliando le farmacopee dei secoli passati non è raro imbattersi nell'utilizzo terapeutico di sostanze oggi ritenute inefficaci, bizzarre, nocive, o addirittura letali; e il ricorso al petrolio come rimedio cutaneo era un rischio tutto sommato tollerabile, per un'epoca in cui ai malati di sifilide si prescrivevano fumigagioni, unzioni, e «salivazioni» a base di mercurio (che era senz'altro efficace nel debellare il morbo, ma spesso aveva lo sgradevole effetto collaterale di debellare anche il paziente).

Quale che ne fosse l'utilizzo, fin dai tempi antichi le salse non passarono inosservate. Già la Naturalis Historia di Plinio narra d'una vera e propria eruzione a cui, nel 91 a.C., assistettero molti viaggiatori in transito lungo la Via Emilia. Fu però con l'affermarsi della filosofia naturale, nel tardo Rinascimento e in Età Moderna, che le indagini si fecero via via più numerose ed approfondite. Furono soprattutto i medici a studiare le salse, spinti com'erano dall'incessante bisogno di trovare nuovi rimedi per gli infiniti malanni che affliggevano il corpo umano. Ma molti altri personaggi (speziali, eruditi, ciarlatani di vario genere, alchimisti, semplici curiosi, ecc.) esplorarono quei brulli terreni, prelevando campioni di fango e d'olio di sasso e cercando di carpirne le caratteristiche e i possibili utilizzi.

I processi geologici all'origine delle salse - così dette per la loro caratteristica salinità, pari a $1 / 2-1 / 3$ di quella marina - sembrano oggi ben compresi. Queste miscele fangose, che scaturiscono dal terreno a bassa temperatura, si seccano vicino ai crateri e si accumulano a formare vulcanetti di pochi metri d'altezza (Fig. 1). La fuoriuscita del gas è provocata dai lenti, ma costanti movimenti della crosta terrestre, che di volta in volta causano l'apertura o la compressione delle sacche sotterranee che contengono la miscela: i vulcani crescono in altezza se il fango esce più velocemente di quanto non ne porti via l'acqua piovana, mentre, in caso contrario, diventano sempre più piccoli. Da qui un'altra tipica caratteristica di questi fenomeni: l'estrema instabilità, che nel giro di pochi anni può aumentarne l'estensione di molto o di molto diminuirla, in alcuni casi fino alla totale sparizione.

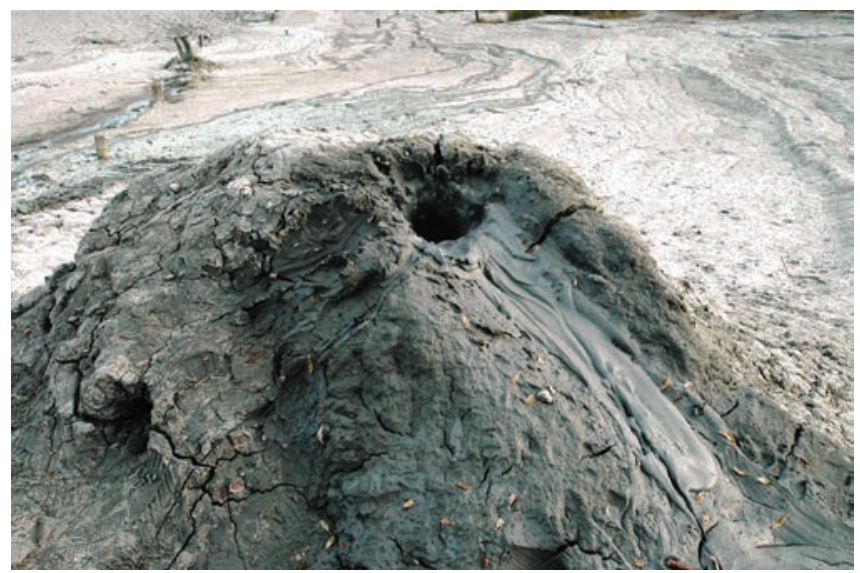

Fig. 1 - Salse di Regnano. Un cratere secondario (foto di Stefano Meloni).

Stando alle testimonianze storiche, le salse emiliane erano un tempo molto più attive. Sono comunque ancora ben visibili in diverse località, in particolare nel Reggiano e nel Modenese. Famose sono quelle di Nirano e Montegibbio (o Monte Zibio), vicino a Sassuolo, e di Regnano, queste ultime in provincia di Reggio Emilia, fra le più appariscenti nel loro genere. Le fuoriuscite di gas avvengono di solito a intervalli di pochi secondi; per intensità e frequenza non sono costanti nel tempo, e passano da fasi di calma a episodi d'emissione più abbondante. Fra gli autori che ne «scrissero le virtù» possiamo annoverare il letterato Francesco Ariosto (1415-1484), i medici Andrea Cesalpino (1519-1603), Gabriele Falloppio (1523-1562) e Andrea Bacci (1524-1600), e persino il medico e filosofo Girolamo Cardano (1501-1576), che vi dedicò alcuni paragrafi nel quinto libro del suo De subtilitate (Cardano 1550). Ma è soltanto a partire dall'ultimo decennio del XVII secolo che troviamo le descrizioni più esaustive e sperimentalmente ben definite, stese dal medico Bernardino Ramazzini (1633-1714) e, non a caso, dai due maggiori naturalisti italiani settecenteschi: Antonio Vallisneri (1661-1730) e Lazzaro Spallanzani (1729-1799).

Vallisneri visitò le salse a partire dal 1694. Si concentrò in particolare su quelle di Querciola, nel territorio di Regnano, annotando l'esperienza nei suoi Quaderni di osservazioni:

«Fui a vedere la salsa di Querzola. Questa in due lochi bolliva, come pentola al foco, e di quando in quando gettava all'aria spruzzi di fango. [...] Poco lungi dal sito de' bollori vi 
sono due sorgenti d'acqua limpida, e cristallina, e mutando loco quelli, ancor l'acqua muta loco. [...] Anticamente era piccolissima, e appena cognita, ora è grandissima, ed occupa di spazio un buon tratto del monte. Alle volte ha spruzzata terra, e sassi sin sopra una casa lungi un tiro di pistola, ed una volta fra l'altre furono necessitati a fuggire, tremando, e muggendo il suolo, di modo, che la casa in varii lochi sdrucì. [.... Quella terra, che vomita fuori è bonissima per esiccare i tumori particolarmente delle gambe. Ne' tempi umidi, e quando il tempo vuol far mutazione di buono in cattivo, bolle, e romoreggia più del solito, e a noi da Scandiano rassembra il tuono, quando è più cupo, e lontano, e facilmente inganna. Dicono esservene un'altra verso i nostri Appenini, ma più grande, siccome asseriscono essere più grande anche quella di Montezibio di Sassolo» (Vallisneri 2004; Luzzini 2013).

Le dettagliatissime descrizioni di Spallanzani (che di Vallisneri fu assiduo lettore) risalgono invece all'ultimo decennio del XVIII secolo, e si vede. In esse si ricorre a termini, criteri e tecniche d'indagine assai più precisi e definiti, rivelatori dell'evoluzione che interessò la ricerca sperimentale nel corso del Settecento. L'autore analizza sia i fanghi che l'«aria infiammabile di Querzuola», che attesta non contenere «gaz oxigene»: pochissima è invece l'«aria fissa [anidride carbonical», come «due importanti esperimenti, l'uno con l'acqua di calce [una soluzione d'idrossido di calcio], l'altro con la tintura di turnasole», hanno dimostrato.

«Alla distanza di 1/4 di miglio dalla salsa, nel discendere ho preso un pezzo di terra da un campo lavorativo, che essa pure era bianca, e sottoposta all'acido muriatico ha fatto molta, e subita effervescenza, lasciando però molta terra in fondo: feltrato il liquido ed accresciuto con acqua stillata, questo ha fatto un bell'azzurro di Berlino [precipitato di ferrocianuro ferrico], versando in esso l'alkali flogisticato [ferrocianuro di potassio]: questa terra contiene adunque del ferro» (Spallanzani 2009).

Gli appunti di Spallanzani confermano la forte attività delle salse settecentesche, che, a detta dei testimoni, poteva sortire effetti spettacolari. Durante le eruzioni più violente i getti di fango potevano raggiungere l'altezza delle chiome degli alberi vicini, e si formavano profonde fenditure verticali nel terreno. In uno degli episodi più significativi, accuratamente descritto nel 1796 dal medico Domenico Gentili (Gentili 1833), al termine dell'eruzione la massa di fango collassò e provocò una frana nei campi sottostanti.

Le ultime due eruzioni degne di nota, stando alle cronache, avvennero a Regnano nel 1915 (durata 15 giorni) e nel 1932. Ben diversa è la situazione attuale, che vede le salse attraversare una fase di quiescenza e di conseguente riduzione della superficie (Fig. 2). Il loro caratteristico grigiore non cessa però di balzare all'occhio di quanti, avventurandosi tra le dolci colline dell'Appennino Emiliano, s'imbattono improvvisamente in un paesaggio più lunare che campestre; e subiscono il fascino dello stesso spettacolo che, nel corso dei secoli, calamitò l'attenzione di schiere di filosofi.

\section{BIBLIOGRAFIA}

Cardano G. (1550). De subtilitate libri 21. Apud Ioh. Petreium, Norimbergae.

Gentili D. (1833). Osservazioni fatte dal Dottore Domenico Gentili sulla salsa di Querzuola e segnatamente sull'eruzione del 22 aprile 1796. Notizie biografiche, e letterarie degli scrittori dello Stato Estense 1833, 1:275-283

Luzzini F. (2013). Il miracolo inutile. Antonio Vallisneri e le scienze della Terra in Europa tra XVII e XVIII secolo. Olschki, Firenze, pp. 74-77, 95 .

Spallanzani L. (2009). Edizione Nazionale delle Opere di Lazzaro Spallanzani - Opere edite non direttamente dall'Autore. Volume IV, a cura di Paola Manzini. Mucchi Editore, Modena, pp. 157-158, 160.

Vallisneri A. (1728). Raccolta di varie Osservazioni, spettanti all'Istoria Medica, e Naturale. Per Domenico Lovisa, Venezia, p. 67.

Vallisneri A. (2004). Quaderni di osservazioni. Volume I, a cura di Concetta Pennuto. Olschki, Firenze, pp. 40-41

www.comune.viano.re.it

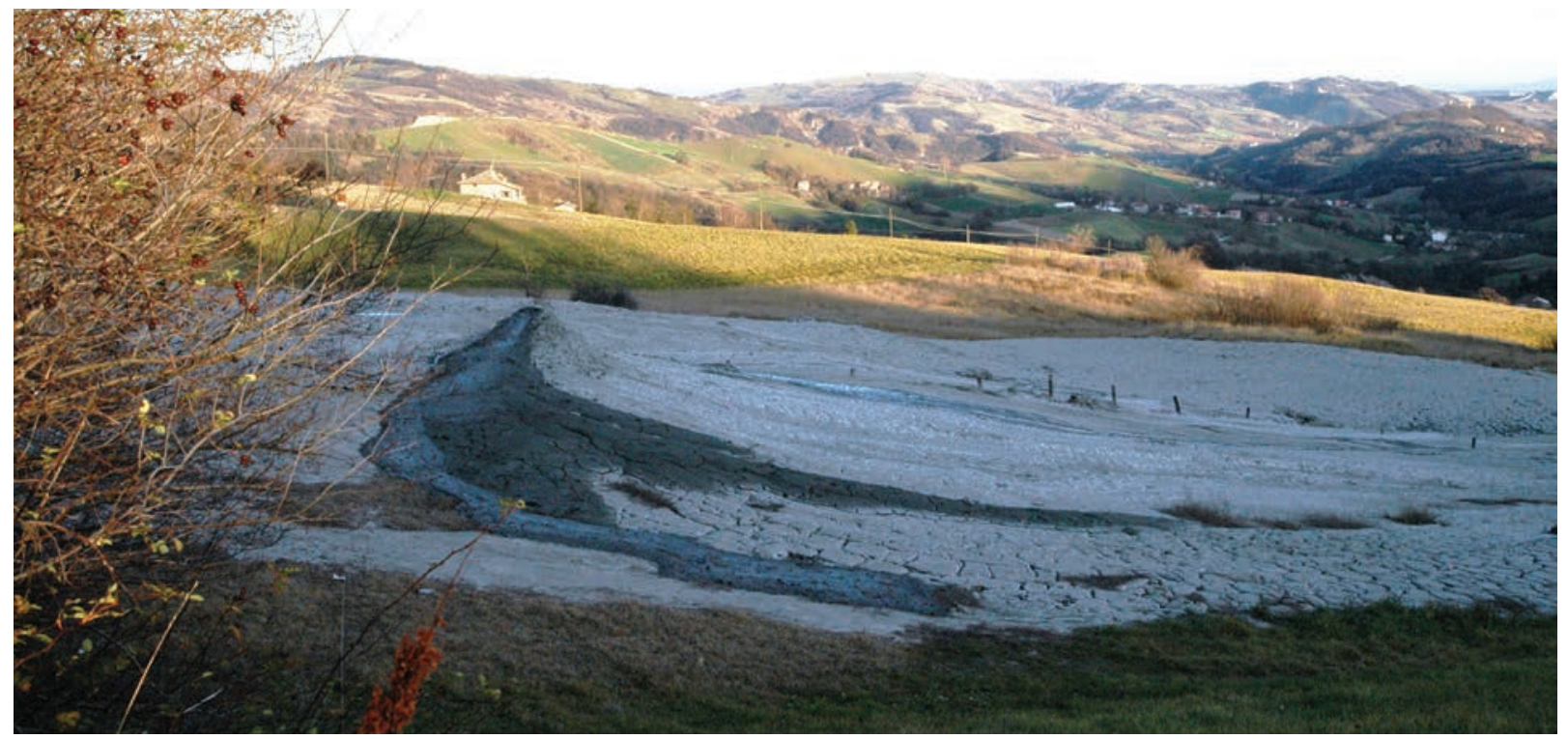

Fig. 2 - L'area coperta dalle salse di Regnano (foto di Stefano Meloni). 